\title{
Nuclear cardiology: an overview of radioisotope techniques used in the diagnostic workup of cardiovascular disorders
}

\author{
1 Nuclear Medicine Department, Greater Poland Cancer Centre, Poznań, Poland \\ 2 1st Department of Cardiology, University of Medical Sciences, Poznań, Poland \\ 3 Nuclear Medicine Department, Medical University of Lublin, Lublin, Poland \\ 4 Department of Electroradiology, University of Medical Science, Poznań, Poland \\ 5 Medical Physics Department, Greater Poland Cancer Centre, Poznań, Poland
}

Paulina Cegła', Aleksandra Ciepłucha², Marcin Pachowicz ${ }^{3}$, Beata Chrapko ${ }^{3}$, Tomasz Piotrowski ${ }^{4}, 5$, Maciej Lesiak ${ }^{2}$

\section{KEY WORDS}

cardiac metabolism, cardiac perfusion, nuclear cardiology, positron emission tomography, single-photon emission computed tomography
Correspondence to: Aleksandra Ciepłucha, MD, PhD, 1st Departmentof Cardiology, University of Medical Sciences, ul. Długa 1-2, 61-848 Poznań, Poland, phone: +48618549146, email: aleksandra.cieplucha@skpp.edu.pl Received: February 24, 2020 Revision accepted: May 25, 2020 Published online: May 28, 2020. Kardiol Pol. 2020; 78 (6): 520-528 doi:10.33963/KP.15396

Copyright by the Author(s), 2020

\begin{abstract}
Cardiovascular diseases are the most common cause of death in patients over 60 years old. Pivotal imaging modalities in cardiac diagnostic workup are echocardiography, magnetic resonance, multi-row detector computed tomography, coronary angiography, and radioisotope tests. In this study, we summarize the techniques of nuclear medicine (positron emission tomography, single-photon emission computed tomography, radionuclide ventriculography) that could be implemented in the cardiovascular diagnostic algorithms. Despite being acknowledged in a few cardiology guidelines, these imaging methods are still underestimated by practitioners. Nevertheless, noninvasive diagnostic tools are of increasing potential and should be implemented whenever possible. We discuss the usefulness of particular techniques in the management of patients with obstructive and nonobstructive coronary artery disease, including assessment of myocardial perfusion, contractility, viability, and detection of unstable atherosclerotic plaques. Radioisotope imaging can also be valuable in the diagnostic workup of infective endocarditis, as well as cardiac sarcoidosis and amyloidosis. Apart from theoretical principles of nuclear cardiology, we also provide 3 case reports illustrating a practical implementation of these imaging modalities.
\end{abstract}

Introduction Cardiovascular diseases are the leading cause of death in patients over 60 years old in Poland. 'Therefore, there is increased interest in diagnostic techniques that allow for a noninvasive detection of cardiovascular diseases and are helpful in the decision-making process for optimal management.

One of the most frequent noninvasive imaging methods used in cardiology is echocardiography. It allows for visualization of cardiac anatomy and function, both at rest and under stress conditions. Other noninvasive methods used in cardiac imaging are multi-row detector computed tomography (MDCT) and cardiac magnetic resonance (CMR). Apart from the multidimensional visualization of the heart anatomy, MDCT is particularly useful in the calcification assessment, whereas CMR-with better spatial resolution than $\mathrm{MDCT}$ - in the myocardial viability. ${ }^{2}$

In contrast to the methods that focus on cardiac anatomy listed above, the imaging tools used in nuclear cardiology are mostly dedicated to the functional assessment based on the uptake of radioisotopes by the cardiac structures. This enables to evaluate the myocardial perfusion, metabolism and viability, adrenergic innervation, radionuclide ventriculography, and intracardiac leakage (both left-to-right and right-toleft). Although nuclear cardiology methods are acknowledged and recommended by experts in the European Society of Cardiology (ESC) guidelines, ${ }^{3,4}$ its clinical value seems to be still underpowered most probably by limited availability 


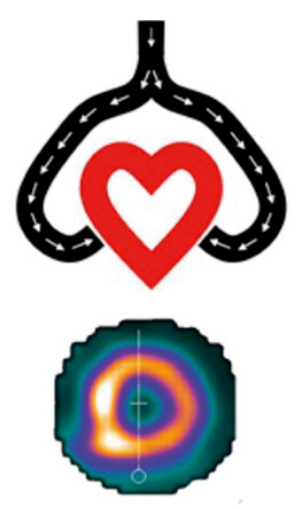

Rest

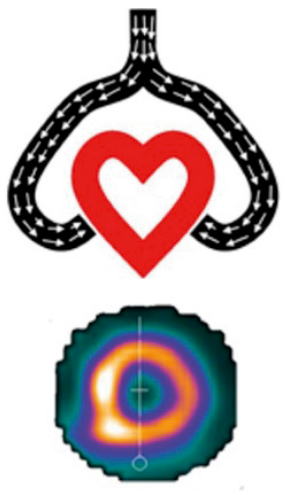

Stress
FIGURE 1 Interpretation of basic myocardial perfusion scan: normal result. At rest, coronary blood flow is even, so radiotracer uptake in the myocardium appears to be homogenous. During stress conditions, coronary blood flow increases and, if there is no obstruction related to coronary artery stenosis, radiotracer concentration in the myocardium remains uniform.

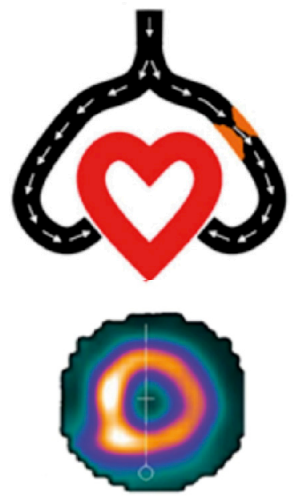

Rest

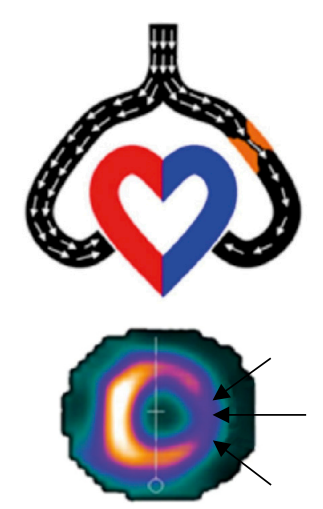

Stress
FIGURE 2 Interpretation of basic myocardial perfusion scan: an abnormal result related to CAD. Despite coronary artery stenosis, coronary blood flow is still unaltered at rest. During stress conditions, coronary blood flow rises, but due to obstruction caused by the atherosclerotic plaque, the area of the myocardium supplied by the stenotic artery receives less blood than other areas, so more radiotracer gets accumulated in healthy areas.

and inadequate experience of cardiologists in the real-life setting.

This article aims to summarize the acknowledged methods of nuclear cardiology as well as its novel diagnostic modalities potentially useful in noninvasive clinical management. We also aim to present practical aspects of nuclear medicine by describing 3 patients undergoing selected procedures that were helpful in the decision-making process.

Myocardial perfusion scan Myocardial perfusion scan (MPS) is one of the most widely used techniques in nuclear cardiology. It allows for detection of coronary artery disease (CAD) and assessment of the functional significance of previously diagnosed lesions in coronary vessels before the introduction of invasive management. ${ }^{5}$ Myocardial perfusion scan can be performed using single-photon emission computed tomography (SPECT) and positron emission tomography (PET). In both cases, hybrid imaging might be performed, that is, complementing the SPECT or PET with computed tomography (CT) ${ }^{6}$

The most popular tracer used in MPS is sestamibi (MIBI) labeled with technetium 99m ( ${ }^{99 \mathrm{~m} T c}$; INN, technetium $99 \mathrm{~m}$ sestamibi). The ${ }^{99 \mathrm{~m} T \mathrm{Tc}-\mathrm{MIBI}}$ chelate is administered intravenously in patients undergoing SPECT imaging. ${ }^{6}$ Intracellularly, MIBI accumulates in the mitochondrial cristae; therefore, its uptake is dependent on the function of the cell organelle. Methoxyisobutylisonitrile is a molecule with positive electrical potential, which in combination with the negative electrical potential of the mitochondrial membrane, allows the accumulation of the radioisotope and significantly reduces the reverse diffusion (only $5 \%$ within 6 hours). ${ }^{7}$ However, the ${ }^{99 \mathrm{mTc}-\mathrm{MIBI} \text { is }}$ a nonspecific radiotracer-it accumulates in the muscular tissue, liver, spleen, and thyroid gland, and only $1.5 \%$ of the administered ${ }^{99 \mathrm{~m}}$ Tc-MIBI accumulates in the myocardium. $^{8}$

Myocardial perfusion scan consists of 2 parts: rest and stress. The physical stress (treadmill or cycle ergometer) or pharmacological agent, that is, dobutamine, adenosine, dipyridamole (adenosine disintegration inhibitor), or regadenoson (selective adenosine $A_{2 A}$ receptor agonist) may be used during the test. Agents causing coronary dilation and passive myocardial congestion (adenosine, dipyridamole, and regadenoson) are preferred over dobutamine. Regadenoson is characterized by a better safety profile, particularly in patients with bronchial asthma or chronic obstructive pulmonary disease. ${ }^{7}$ The nuclear medicine specialist chooses the method and order of the study stages based on the individual medical history, available data, and the results of previous examinations.

The patient should be fasting for at least 8 hours before the planned MPS test. Unless there are contraindications, medications that may affect the course of the exercise test ( $\beta$-blockers, blockers of calcium channels, and long-acting nitrates) should be discontinued 48 hours before the examination. Long-acting theophylline derivatives (adenosine antagonist) as well as coffee and tea (as a natural source of theophylline) should be avoided.

Evaluation of myocardial perfusion An intravenously injected radiotracer accumulates in the myocardium proportionally to the blood flow (Supplementary material, Figure S1). The ${ }^{15} \mathrm{O}$-labeled water $\left(\mathrm{H}_{2}{ }^{15} \mathrm{O}\right)$ freely diffuses through the cell membrane and therefore is characterized by a linear relationship between the blood flow and radiotracer extraction. In contrast, the uptake of other radioisotopes decreases with increasing flow. This phenomenon is called a "roll-off" effect and may lead to misinterpretation of 
the result because small stenosis of the coronary artery may not show a significant change in radiotracer accumulation and thus may not be detected. ${ }^{9}$ The basis for interpretation of MPS studies is demonstrated in FIGURES 1 and 2.

Normal radiotracer distribution in myocardium indicates a very low probability of myocardial ischemia. If there is an area of decreased radiotracer accumulation during the stress study, which entirely or partially resolves during rest, it is a reversible perfusion defect. It indicates stress-related ischemia and is associated with hemodynamically significant stenosis of a coronary artery supplying a particular myocardial region. If there is no difference in the stress and rest studies and decreased radiotracer accumulation remains the same, it is a fixed perfusion defect. This is typically related to a previous ischemic episode and may indicate both postinfarction scars as well as hibernated myocardium. The molecular mechanism behind cellular uptake of MIBI is strictly related to mitochondrial function and integrity; therefore, its distribution may be insufficient to distinguish between these 2 conditions. Inflammatory diseases, including myocarditis, may also alter perfusion due to local inflammation and necrosis. The absence of MIBI uptake requires further verification with myocardial viability PET scans with glucose analog radiolabeled with fluorine $18\left({ }^{18} \mathrm{~F}\right),{ }^{18} \mathrm{~F}$-fluorodeoxyglucose $\left({ }^{18} \mathrm{~F}-\mathrm{FDG}\right) .{ }^{10,11}$ MPS with ${ }^{99 \mathrm{~m} T c-M I B I}$ has approximately $87 \%$ sensitivity and $73 \%$ specificity. ${ }^{6}$

Myocardial perfusion SPECT performed with electrocardiography (ECG)-gating provides additional data on the left ventricular function (end-diastolic volume, end-systolic volume, ejection fraction, myocardium mass, as well as regional wall motion). Functional data delivered by gated SPECT may improve the detection of 3-vessel disease or left main stem disease. If needed, the assessment of myocardial stunning may be performed, with a temporary reduction of the myocardial contractility observed only during the stress stage, but not at rest. ${ }^{12,13}$

Gated SPECT has also been used in the assessment of left ventricular contraction synchrony. This method enables precise tracing of myocardial activation and may detect disturbances in this process associated with mechanical contraction asynchrony. Experts postulate that mechanical asynchrony is related to left ventricular dysfunction (ejection fraction reduction) to a greater extent than perfusion defect size..$^{9,14,15}$

PET has superior image quality compared with SPECT. It is due to more favorable radiotracer properties used in PET and more precise spatial resolution (4-7 $\mathrm{mm}$ for $\mathrm{PET}$ vs $12-15 \mathrm{~mm}$ for SPECT), which allows for detecting even minor perfusion defects. In addition to the better spatial resolution, PET has a more precise temporal resolution. This feature enables a more precise evaluation of radiotracer activity in the coronary arteries and myocardium. Moreover, in PET imaging, absolute myocardial perfusion may be calculated (milliliters per gram of tissue per minute), whereas in SPECT, only the relative myocardial perfusion can be assessed. Therefore, ${ }^{18} \mathrm{~F}-\mathrm{FDG}$ -PET is believed to be a more accurate diagnostic tool for the assessment of myocardial perfusion. ${ }^{16}$

Myocardial viability assessment Nuclear imaging with PET and SPECT is particularly useful in cardiac muscle viability assessment. According to the recent guidelines on myocardial revascularization, myocardial viability assessment is crucial in patients with chronic total occlusion of coronary arteries and severely depressed left ventricular systolic function. Preserved viability is considered an indication for coronary artery bypass grafting or percutaneous coronary intervention before cardiac transplantation or implantation of devices that mechanically assist circulation. ${ }^{3}$ As mentioned above, the MPS performed with SPECT does not allow for differentiation between post-infarction scar and hibernated myocardium. This is caused by the fact that ${ }^{99 \mathrm{~m}}$ Tc-MIBI accumulates neither within the scar nor in the hibernated cardiomyocytes, as they both include a mitochondrial dysfunction that disables the uptake of the radiotracer. Nonetheless, as hibernated cardiomyocytes still use anaerobic metabolism and utilize glucose, they can accumulate ${ }^{18} \mathrm{~F}-\mathrm{FDG}$. The comparison of perfusion and metabolism ( ${ }^{99 \mathrm{~m}} \mathrm{Tc}-\mathrm{MIBI}$ and ${ }^{18} \mathrm{~F}-\mathrm{FDG}$ uptake) enables the identification of viable myocardium. If ${ }^{18} \mathrm{~F}$-FDG uptake is observed in the areas representing weak or absent MIBI uptake, it is indicative of hibernation and the viable myocardium (perfusion-metabolism mismatch). The absence of both MIBI and ${ }^{18}$ F-FDG uptake confirms a scar (perfusion-metabolism match; Figure 3, tABLE 1). ${ }^{17-19}$ Images obtained by ${ }^{18}$ F-FDG-PET enable determining the viability of dysfunctional myocardial regions (sensitivity, $88 \%$; specificity, $73 \%$; positive predictive value, $82 \%$; negative predictive value, $83 \%$ ) and predict recovery of global contractile function after revascularization. ${ }^{20}$

Coronary microvascular dysfunction Coronary microvascular disease (MVD) includes functional changes of coronary microvasculature that disable proper vasodilatation and augmentation of coronary blood flow required during exercise. ${ }^{21}$ Coronary flow reserve (CFR) measured by PET (defined as the ratio between myocardial blood flow calculated in $\mathrm{ml} / \mathrm{min} / \mathrm{g}$ at stress and at rest) quantifies a general coronary vasomotor function by integrating hemodynamic effect of epicardial stenosis, diffuse atherosclerosis, and MVD. Depending on the study protocol, normal values of CFR range between 1.4 to 2.7. PET-derived CFR measurements correlate with the biomarkers characteristic of MVD and 

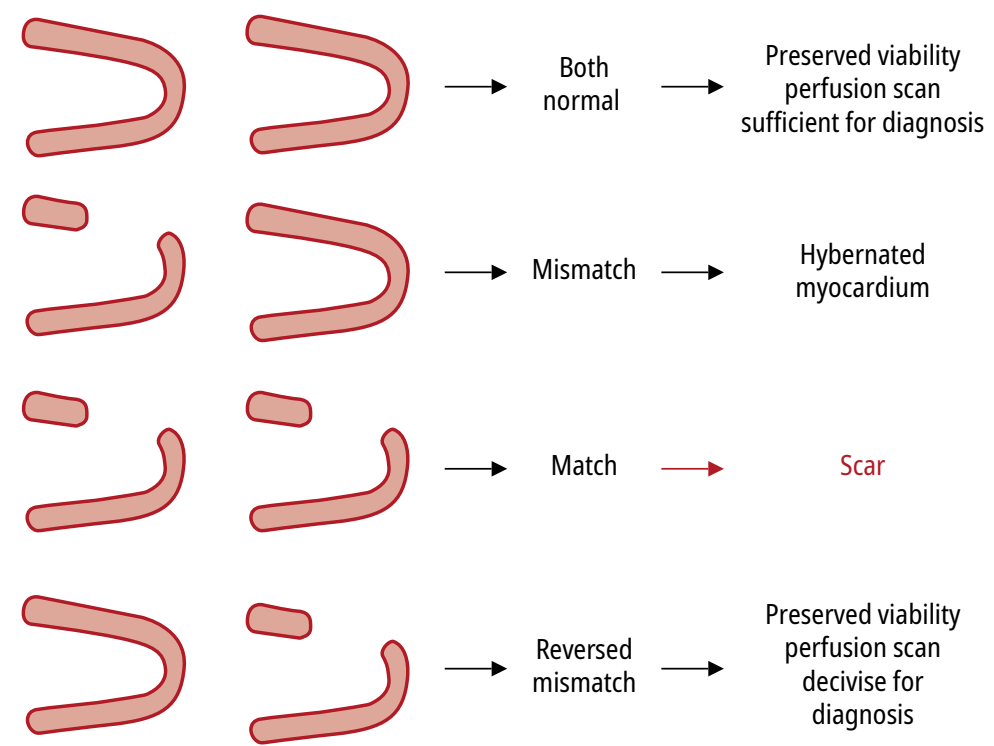

FIGURE 3 Interpretation of myocardial viability in single-photon emission positron

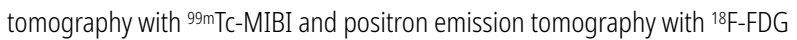

TABLE 1 Basic patterns of interpretation of myocardial viability (based on Kobylecka et al ${ }^{19}$ )

\begin{tabular}{llll} 
Parameter & Standard & Hibernation & Necrosis \\
Perfusion & Present & Absent & Absent \\
\hline Glucose metabolism & Present & Present & Absent \\
\hline Ejection fraction & Present & Absent & Absent \\
\hline
\end{tabular}

may enhance understanding and better management of symptomatic patients. This is particularly helpful in risk stratification, since MVD is common among patients with chest tightness, and a long-term prognosis in MVD is similarly poor when compared with patients with obstructive coronary disease. ${ }^{22-24}$

Unstable atherosclerotic plaques evaluation Unstable atherosclerotic plaque is a plaque that has a large necrotic lipid core, and the thickness of the connective tissue cap does not exceed $65 \mu \mathrm{m}$. Within the plaque, there is an enhanced inflammation dominated by macrophages and T cells. Neovascularization and hemorrhages inside the plaque are also very often observed. ${ }^{25}$ Because of high glucose consumption in vascular macrophages, ${ }^{18} \mathrm{~F}-\mathrm{FDG}$-PET/CT has been used to image inflammatory lesions for several years. An association between the ${ }^{18}$ F-FDG uptake, macrophage burden, and gene expression of CD68 was also confirmed in the study evaluating atherosclerotic plaques in the carotid arteries. ${ }^{26}$ Subsequently, a study by Tarkin et $\mathrm{al}^{27}$ proved superiority of gallium-68-labeled DOTATATE ( ${ }^{68} \mathrm{Ga}$-DOTATATE) over ${ }^{18} \mathrm{~F}$-FDG in differentiating stable and unstable coronary plaques found in CT imaging, as higher uptake of ${ }^{68} \mathrm{Ga}$-DOTATATE was observed in culprit coronary and carotid lesions. It was confirmed by histopathological examinations that ${ }^{68} \mathrm{Ga}$-DOTATATE was present in CD68-positive, macrophage-rich carotid plaques.

Studies have shown that PET/CT scan with sodium fluoride $\left({ }^{18} \mathrm{~F}-\mathrm{NaF}\right)$, used in musculoskeletal system diseases, may be even more beneficial in the assessment of atherosclerotic plaque biology. An increased accumulation was seen in ruptured and high-risk plaques with significantly higher sensitivity than ${ }^{18} \mathrm{~F}-\mathrm{FDG}$ in terms of coronary artery atherosclerosis, due to lack of physiological uptake of ${ }^{18} \mathrm{~F}-\mathrm{NaF}$ by the myocardium. ${ }^{28,29}$ In assessing unstable atherosclerotic plaque, ${ }^{68} \mathrm{Ga}$-DOTATATE- and ${ }^{18} \mathrm{~F}-\mathrm{NaF}$-PET are superior to ${ }^{18} \mathrm{~F}-\mathrm{FDG}-\mathrm{PET}$ studies.

Radionuclide ventriculography Radionuclide ventriculography is used in the assessment of left ventricular function. It utilizes ${ }^{99 \mathrm{~m} T c}$ that bounds to hemoglobin, producing "radiolabeled blood pool." 30,31

When ECG gating is used, changes in blood pool radioactivity can be tracked, both with SPECT or dynamic methods and it enables to calculate the left ventricular volumes and ejection fraction (FIGURE 4). SPECT imaging also demonstrates the regional wall motion by tracking the endocardium movement. Radionuclide ventriculography is one of the most accurate techniques to evaluate left ventricular ejection fraction. Due to anatomical conditions, evaluation of the right ventricle is much more complicated and is not commonly used. ${ }^{30}$

Adrenergic system imaging Catecholamine analog-metaiodobenzylguanidine (mIBG) labeled with iodine 123 ( $\left.\mathrm{m}^{123} \mathrm{IBG}\right)$ - enables to assess of the function of the cardiac adrenergic system. The analysis is based on the assessment of presynaptic $\mathrm{m}^{123}$ IBG uptake by the cardiac adrenergic system. The measurements are performed at 2 time points, enabling visualization of a flushing effect. Obtained values provide information about the integrity of the cardiac adrenergic system and its activity. The test is used primarily in patients with heart failure as it may help to predict the response to the applied treatment, that is, cardiac resynchronization therapy or ablation of atrial fibrillation. ${ }^{32-37}$

Infective endocarditis imaging In recent years, the occurrence of infective endocarditis (IE) has increased. According to the ESC guidelines, the gold standard for imaging patients with IE remains echocardiography. ${ }^{4}$ However, conventional imaging techniques and microbiology results are inconclusive in patients after implantation of prosthetic valves and cardiac 


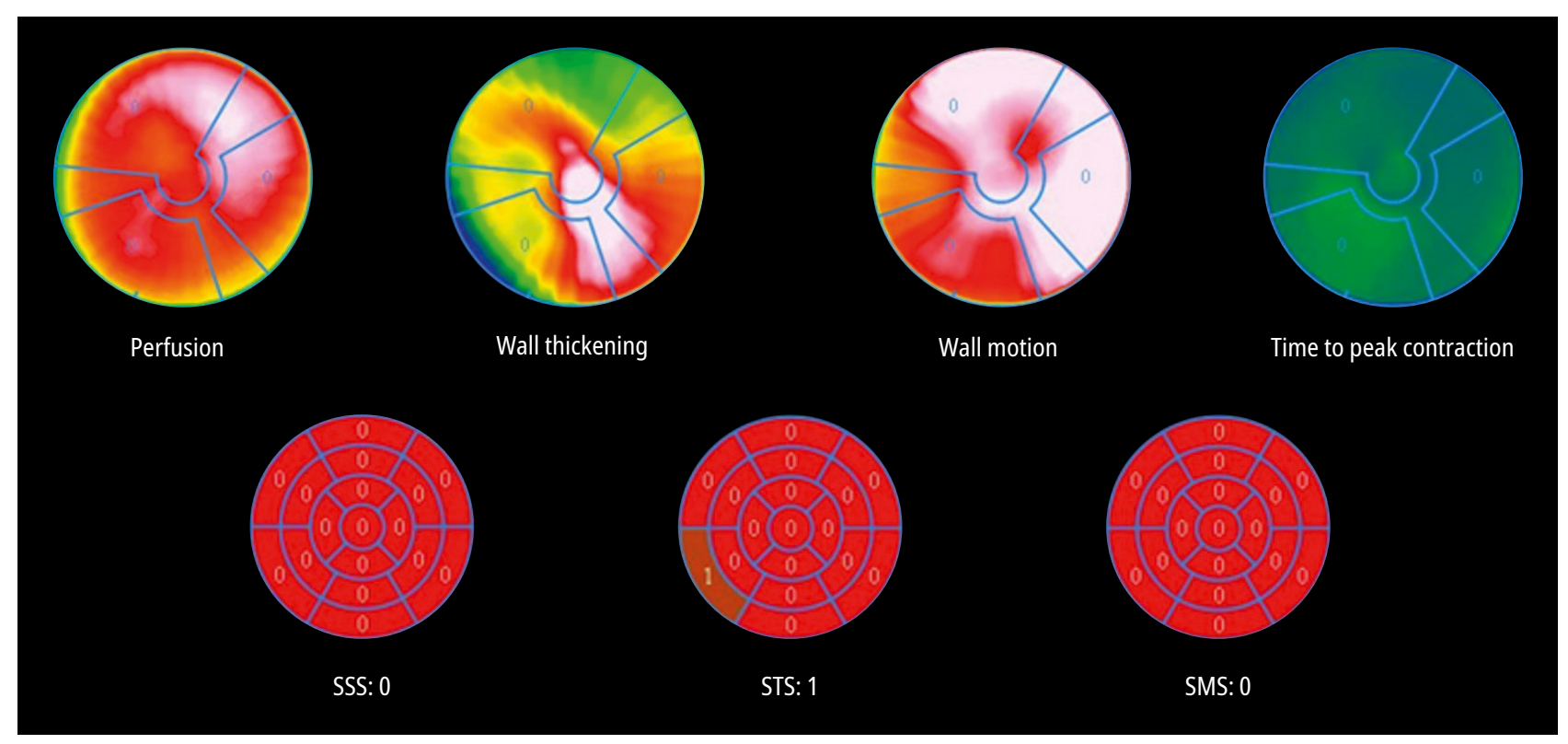

\begin{tabular}{llllll}
\hline Perf. & 0: normal & 1: equivocal & 2: abnormal & 3: severe & 4: absent \\
\hline Thick. & 0: normal & 1: equivocal & 2: abnormal & 3: none & \\
\hline Mol. & 0: normal & 1: equivocal & 2: hypokinetic & 3: akinetic & 4: dyskinetic \\
\hline
\end{tabular}

\begin{tabular}{lllll} 
Region & $\begin{array}{l}\text { Perfusion } \\
\text { extent }\end{array}$ & $\begin{array}{l}\text { Wall thickening } \\
\text { extent }\end{array}$ & $\begin{array}{l}\text { Wall motion } \\
\text { extent }\end{array}$ & $\begin{array}{l}\text { Time to peak } \\
\text { contraction extent }\end{array}$ \\
\hline LAD & $0 \%$ & $0 \%$ & $0 \%$ & $0 \%$ \\
\hline LCX & $0 \%$ & $0 \%$ & $0 \%$ & $0 \%$ \\
\hline RCA & $0 \%$ & $0 \%$ & $0 \%$ & $0 \%$ \\
\hline TOT & $0 \%$ & $0 \%$ & $0 \%$ & $0 \%$ \\
\hline
\end{tabular}

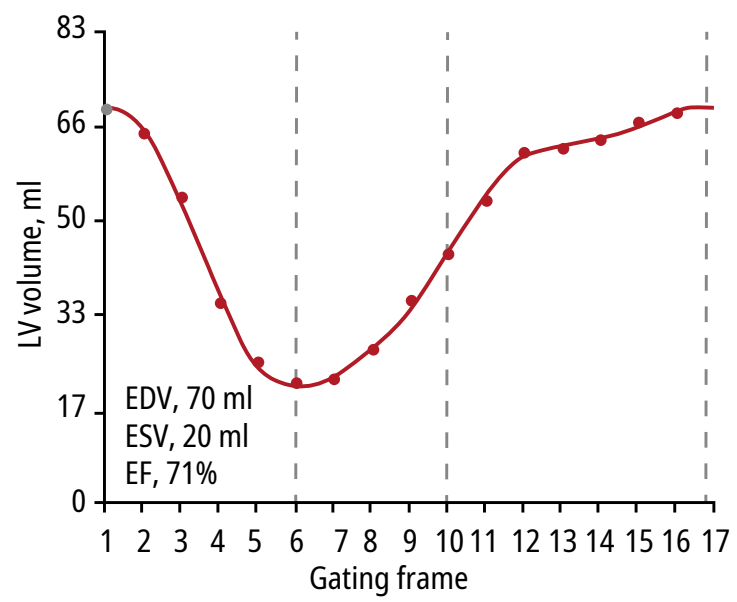

FIGURE 4 Report of left ventricular function indicating normal wall thickening and motion with normal left ventricular volume, ejection fraction, and muscle mass Abbreviations: EDV, end diastolic volume; EF, ejection fraction; ESV, end systolic volume; LAD, left anterior descending; LCX, left circumflex branch; Mol, wall motion; Perf, perfusion; RCA, right coronary artery; Thic, wall thickening; TOT, total; LV, left ventricle

implantable electronic devices (CIEDs). In this setting, SPECT/CT with indium 111 (111 In) or ${ }^{99 \mathrm{~m}} \mathrm{Tc}$ labeled leukocytes is most commonly used. The implementation of SPECT/CT with ${ }^{99 \mathrm{~m} T c}$ labeled hexamethylpropyleneamine oxime ${ }^{99 \mathrm{~m}} \mathrm{Tc}-$ -HMPAO) is particularly useful in patients with a prosthetic valve. It shows an abnormal uptake of radiotracer around the prosthesis in case of endocarditis. Moreover, it is recommended as an additional diagnostic tool (class IIb recommendation) in patients with CIEDs with clinical suspicion of IE, negative echocardiography, and positive results of blood cultures..$^{38-41}$

${ }^{18} \mathrm{~F}$-FDG PET/CT study has been introduced in the modified Duke's criteria for the diagnostic workup of IE. Despite low sensitivity in native valves ( $<40 \%)$, this technique has shown significant accuracy (sensitivity $87 \%$, specificity $92 \%$ ) in prosthetic valve IE or CIEDs. The introduction of this method to the diagnostic workup enhanced the sensitivity of the modified Duke's criteria from $52 \%$ to $91 \%$, and therefore has been incorporated in the diagnostic algorithm proposed in the 2015 ESC guidelines. ${ }^{4}$

A spatial resolution of PET limits the chance of imaging areas smaller than $5 \mathrm{~mm}$. At the same time, due to the high physiological activity of ${ }^{18} \mathrm{~F}-\mathrm{FDG}$, it is impossible to visualize septic emboli in the brain. Moreover, it is unfeasible to distinguish between an aseptic postoperative inflammation and infection in the first 3 months after cardiac surgery. Also ${ }^{18} \mathrm{~F}$-FDG-PET does not differentiate thrombi, soft atherosclerotic plaque, primary cardiac tumors, postoperative inflammatory infiltrates, and reaction to a foreign body. ${ }^{4,42,43}$

Some of the disadvantages of scintigraphy with labeled leukocytes compared with PET/CT are the lower spatial resolution and its limited value in nonbacterial infections (the majority of leukocytes labeled are neutrophils). ${ }^{43}$ 

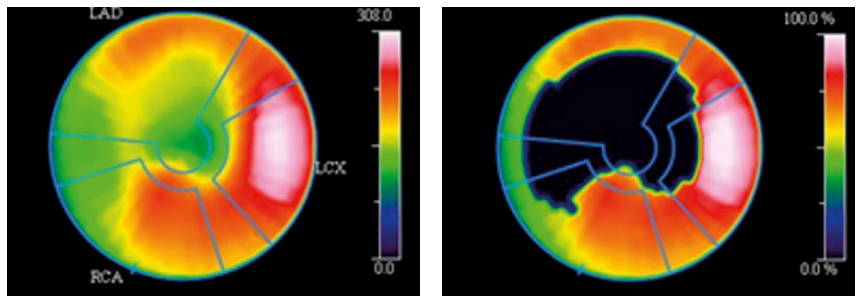

\begin{tabular}{ll} 
Region & Extent \\
\hline LAD & $73 \%$ \\
\hline LCX & $28 \%$ \\
\hline RCA & $16 \%$ \\
\hline TOT & $49 \%$ \\
\hline
\end{tabular}

Perfusion

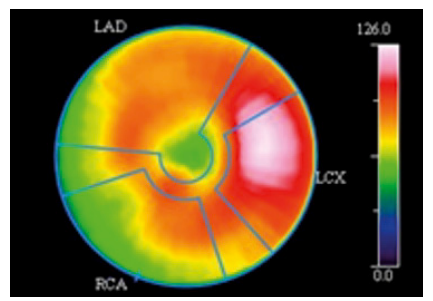

Reversibility

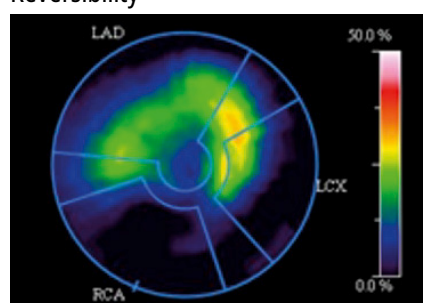

Defect blackout map

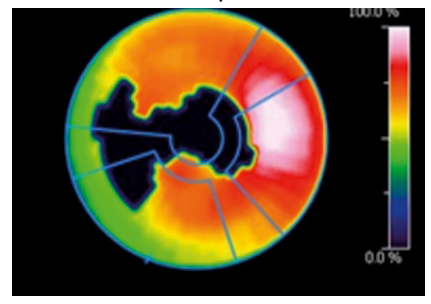

Defect blackout map

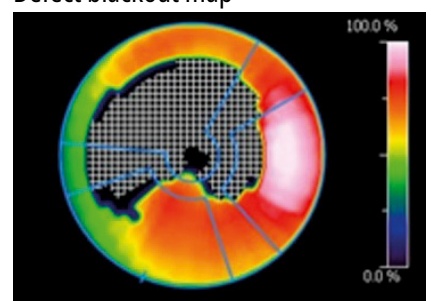

\begin{tabular}{ll} 
Region & Extent \\
\hline LAD & $39 \%$ \\
\hline LCX & $13 \%$ \\
\hline RCA & $17 \%$ \\
\hline TOT & $31 \%$ \\
\hline
\end{tabular}

\begin{tabular}{llll} 
Region & Nml & Fixed & Revers. \\
LAD & $27 \%$ & $6 \%$ & $67 \%$ \\
\hline LCX & $72 \%$ & $2 \%$ & $26 \%$ \\
\hline RCA & $84 \%$ & $10 \%$ & $6 \%$ \\
\hline TOT & $51 \%$ & $5 \%$ & $44 \%$ \\
\hline
\end{tabular}

FIGURE 5 Myocardial perfusion polar map. Top row: stress; middle row: rest; bottom row: reversibility; left column: normalized perfusion; right column: defect blackout maps. Stress images show significantly lower concentration of 99mTC-MIBI in the apex, anterior wall, and septum, as well as apical segment of the lateral wall indicating severe myocardial ischemia. Rest images show significant radiotracer accumulation improvement. Perfusion defect reversibility is observed in nearly entire abnormal region covering $44 \%$ of the left ventricular myocardium.

Abbreviations: nml, normal; revers., reversibility; others see FIGURE 4

Cardiac sarcoidosis Cardiac sarcoidosis (CS) may be an incidental diagnosis, albeit it may lead to multiple complications, including advanced heart block, cardiomyopathy, and arrhythmias. Advanced imaging modalities including CMR or PET show similar accuracy in the diagnostic workup of CS compared with autopsy studies. Combining ${ }^{18}$ F-FDG-PET/CT study with perfusion in SPECT/CT is particularly useful, as the co-occurrence of multiple uptake areas associated with the matching perfusion abnormalities seen in SPECT/ CT gives a high probability of an accurate diagnosis. ${ }^{44}$ There have been attempts to assess the therapeutic and prognostic factors using ${ }^{18} \mathrm{~F}-\mathrm{FDG}-\mathrm{PET} / \mathrm{CT}$. Among 137 patients, a pathologic right ventricular ${ }^{18}$ F-FDG uptake occurred more frequently in patients with cardiovascular events compared with those without such events (46\% vs $6 \%$ ). Another study suggested that high total cardiac metabolic activity (up to $900 \mathrm{MBq}$ of ${ }^{18} \mathrm{~F}-\mathrm{FDG}$ ) in relation with right ventricular uptake of radiotracer were significant risk factors of worse prognosis in patients with $C S,{ }^{45}$ thus, using ${ }^{18} \mathrm{~F}-\mathrm{FDG}-\mathrm{PET}$ is more suitable to assess CS than SPECT/CT imaging.
Cardiac amyloidosis Cardiac amyloidosis is currently diagnosed more frequently than in the past owing to the advanced diagnostic modalities. There are 2 most common types of cardiac amyloidosis: light-chain amyloidosis and transthyretin amyloidosis (ATTR), both with distinct therapeutic management and prognosis. Echocardiography and CMR play a crucial role in the diagnostic workup; however, the differentiation between the subtypes of cardiac amyloidosis remains problematic. Radionuclide scintigraphy with ${ }^{99 \mathrm{~m} T c-l a b e l e d ~ d i p h o s p h o n o p r o p a n o d i c a r b o x-~}$

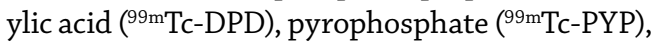
or metylendiphosphonic acid ( $\left.{ }^{99 \mathrm{~m}} \mathrm{Tc}-\mathrm{MDP}\right)$ has been found useful in identifying patients with ATTR. Increased uptake of ${ }^{99 \mathrm{~m} T c-D P D}$ in the myocardium with decreased or absent uptake in the bones suggest the ATTR type. ${ }^{46}$ In a study of 1217 patients who underwent either ${ }^{99 \mathrm{~m}} \mathrm{Tc}-\mathrm{DPD}$ or 99mTc-PYP SPECT, negative results excluded ATTR in $99 \%$, with the specificity of $86 \% .{ }^{47}$ The correlation of positive bone scintigraphy test with a negative result of monoclonal protein in the blood or urine increased specificity to $100 \% .{ }^{48}$

Future directions Radiotracers labeled with ${ }^{18} \mathrm{~F}$, for example, ${ }^{18} \mathrm{~F}$-flurpiridaz used in MPS-PET/CT, 


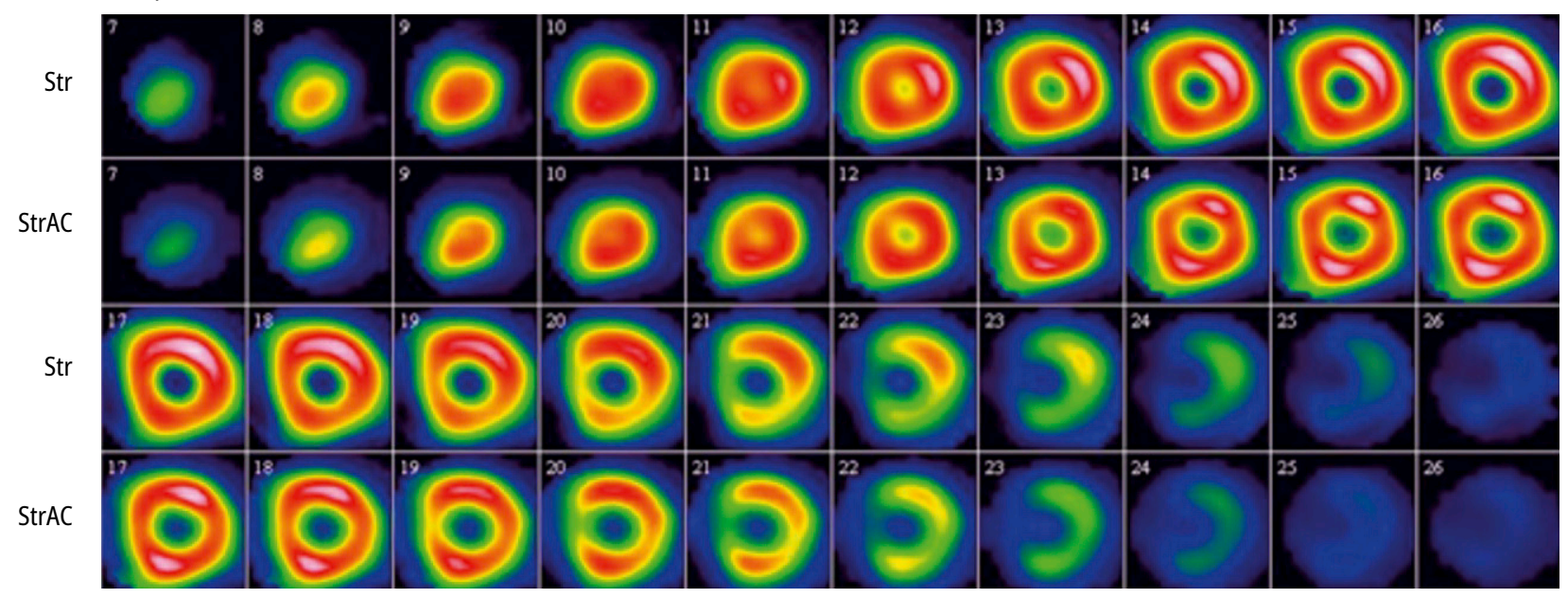

HLA (INF->ANT)

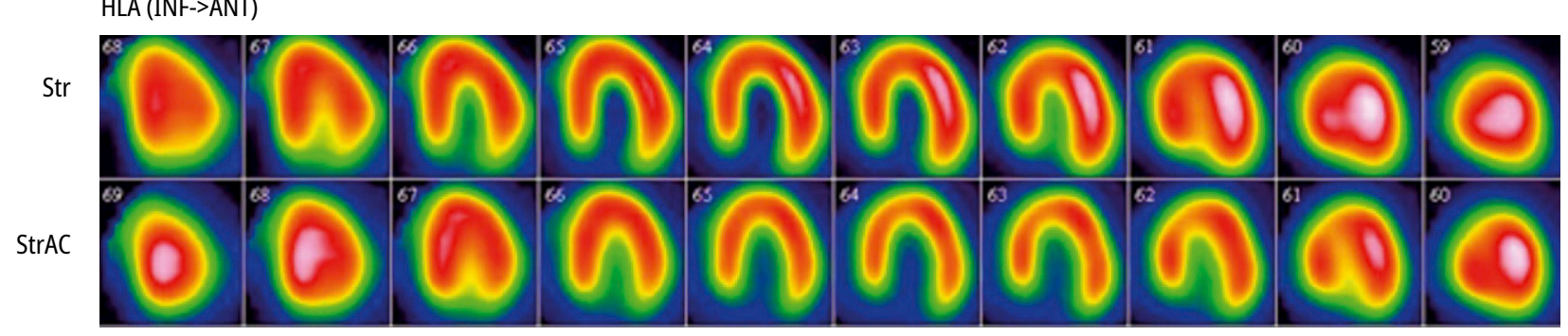

VLA (SEP->LAT)

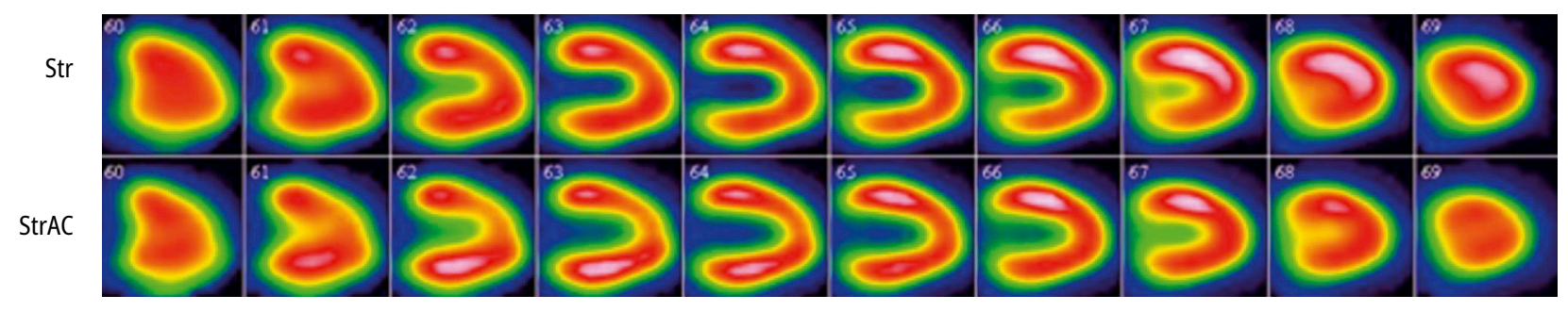

FIGURE 6 Stress perfusion slice-by-slice report: images without and with attenuation correction both present normal myocardial perfusion

Abbreviations: ANT, anterior; HLA, horizontal long axis; INF, inferior; LAT, lateral; SA short axis; SEP, septum; Str, stress; StrAC, stress with attenuation correction; VLA, vertical long axis

can be introduced in the diagnostic evaluation of CAD. In 86 patients who underwent coronary angiography, the sensitivity of PET was higher compared with SPECT (78.8\% to $61.5 \% ; P=0.02)$, with statistically similar specificity (PET, $76.5 \%$ vs SPECT, 73.5\%; $P$ = not significant). The published results showed that ${ }^{18} \mathrm{~F}$-flurpiridaz-PET MPS was safe and qualitatively better than SPECT MPS in the diagnostic workup of CAD. ${ }^{49}$

Recently, Mood et $\mathrm{al}^{50}$ assessed an absolute myocardial blood flow (MBF) and myocardial flow reserve (MFR) in rest and pharmacologic stress in 231 patients. These data were compared with CAD severity quantified by invasive coronary angiography on a per-patient and per-vessel basis. They found that stress MBF per-vessel identified accurately the obstructive disease and, along with MFR, progressively declined with an increasing coronary atherosclerotic burden. The study confirmed the incremental diagnostic value of stress
$\mathrm{MBF}$ and MFR in the diagnostic workup of CAD. Therefore, ${ }^{18} \mathrm{~F}$-flurpiridaz PET is a promising modality in myocardial blood flow measurements.

There have been attempts for noninvasive evaluation of myocardial hypoxia. This phenomenon can be present in ischemic heart disease, including circulation disorders or myocardial hibernation and cardiac hypertrophy. $\mathrm{PET} / \mathrm{CT}$ with ${ }^{18} \mathrm{~F}$-fluoromizonidazole ( ${ }^{18} \mathrm{~F}$-MISO) or ${ }^{18} \mathrm{~F}$-fluoronitroimidazole ( ${ }^{18} \mathrm{~F}$-FAZA) demonstrates a real-time data reflecting the biodistribution of radiopharmaceuticals. ${ }^{51,52}$

Conclusion The use of noninvasive nuclear medicine techniques in the diagnosis of cardiovascular diseases in recent decades has become the subject of greater interest. Improved access to the technologically advanced equipment, as well as the introduction of new radiotracers, allow for addressing multiple clinical questions, 

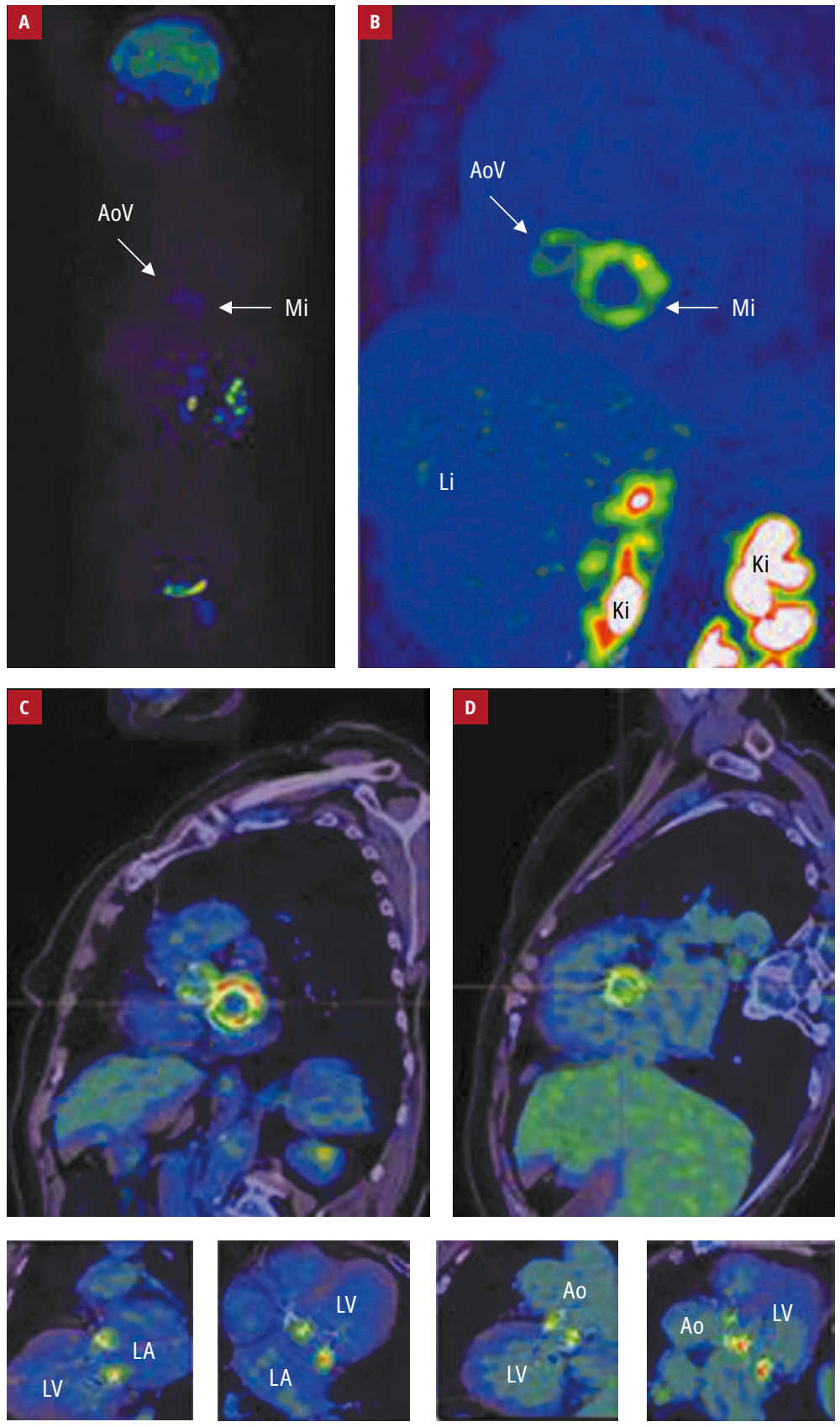

FIGURE 7 Positron emission tomography imaging of the heart: A - whole-body maximum intensity projection indicating abnormal uptake corresponding to both prostheses. Despite that, no obvious abnormal ${ }^{18} \mathrm{~F}-\mathrm{FDG}$ distribution is observed; $\mathbf{B}$ - maximum intensity projection of the chest, left / anterior oblique view, showing ${ }^{18} \mathrm{~F}-\mathrm{FDG}$ uptake mainly in mitral valve ring, lesser accumulation in the aortic valve ring. Positron emission tomography / computed tomography scan: $\mathbf{C}$ - a transmitral plane with auxiliary sections, intense ${ }^{18} \mathrm{~F}-\mathrm{FDG}$ uptake in the mitral valve ring; D - a transaortic plane with auxiliary sections, ${ }^{18} \mathrm{~F}-\mathrm{FDG}$ uptake in aortic valve ring Abbreviations: Ao, aorta; AoV, aortic valve; Ki, kidney; LA, left atrium; Li, liver; LV, left ventricle; $\mathrm{Mi}$, mitral valve pain was originating in the epigastrium and radiating to the interscapular region of the back. The patient presented no CAD risk factors except nicotinism in the past. A routine treadmill stress test and transthoracic echocardiography were normal; subsequent coronary angiography revealed critical stenosis in the proximal left anterior descending artery (FIGURE5).

Case 2 A 60-year-old woman with suspected $\mathrm{CAD}$ and atypical symptoms underwent the treadmill exercise test. It demonstrated ST-segment depression in leads II, III, and aVF suggestive of inferior wall ischemia. In this case, myocardial scintigraphy shown in FIGURE 6 excluded ischemia and proved ECG-stress to be false positive.

Case 3 A 69-year-old asymptomatic woman presented 4 years after a successful mitral and aortic valves replacement (biological prostheses). During routine echocardiographic follow-up, 2 abnormal structures were visualized on the mitral prosthesis. FIGURE 7A-7D demonstrates abnormalities in the ${ }^{18} \mathrm{~F}-\mathrm{FDG}$-PET that was conclusive of active endocarditis on both prostheses.

\section{SUPPLEMENTARY MATERIAL}

Supplementary material is available at www.mp.pl/kardiologiapolska.

\section{ARTICLE INFORMATION}

\section{CONFLICT OF INTEREST None declared.}

OPEN ACCESS This is an Open Access article distributed under the terms of the Creative Commons Attribution-Non Commercial-No Derivatives 4.0 International License (CC BY-NC-ND 4.0), allowing third parties to download articles and share them with others, provided the original work is properly cited, not changed in any way, distributed under the same license, and used for noncommercial purposes only. For commercial use, please contact the journal office at kardiologiapolska@ptkardio.pl.

HOW TO CITE Cegła P, Ciepłucha A, Pachowicz M, et al. Nuclear cardiology: an overview of radioisotope techniques used in the diagnostic workup of cardiovascular disorders. Kardiol Pol. 2020; 78: 520-528. doi:10.33963/KP.15396

\section{REFERENCES}

1 Chmiel J, Książek MK, Stryszak W, et al. Temporal changes in the pattern of invasive angiography use and its outcome in suspected coronary artery disease: implications for patient management and healthcare resources utilization. Adv Interv Cardiol. 2018; 14: 247-257.

2 Davies MJ, Newton JD. Non-invasive imaging in cardiology for the generalist. Br J Hosp Med (Lond). 2017; 78: 392-398.

3 Neumann F-J, Sousa-Uva M, Ahlsson A, et al. ESC/EACTS guidelines on myocardial revascularization [in Polish]. Kardiol Pol. 2018; 76: 1585-1664.

4 Habib G, Lancellotti P, Antunes MJ, et al. 2015 ESC guidelines for the management of infective endocarditis. Kardiol Pol. 2015; 73: 963-1027.

5 Lee WW. Recent advances in nuclear cardiology. Nucl Med Mol Imaging. 2016; 50: 196-206.

6 Birkenfeld B, Listewnik M. Myocardial perfusion. In: Birkenfeld B, Listewnik M, eds. Nuclear Medicine. Molecular Imaging [in Polish]. PUM; 2011: 79-89.

7 Friedman M, Spalding J, Kothari S, et al. Myocardial perfusion imaging laboratory efficiency with the use of regadenoson compared to adenosine and dipyridamole. J Med Econ. 2013; 16: 449-460.

8 Mikołajczak R, Garnuszek P. Radiopharmaceuticals in cardiology. Nucl Med Rev Cent East Eur. 2012; 15: 39-45.

9 Pachowicz M. Left ventricle function and contraction asynchrony assessment in patients with fixed myocardial perfusion defects evaluated with $99 \mathrm{mTc}-$ -sestamibi gated single photon emission computed tomography myocardial perfusion scan [in Polish]. Dissertation. Lublin: Uniwersytet Medyczny w Lublinie, Katedra i Zakład Medycyny Nuklearnej; 2016.
Case presentations Case 1 A 49-year-old man with suspected CAD and atypical clinical presen-

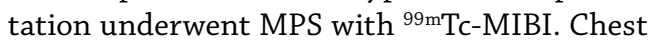


10 Hung GU. Diagnosing CAD: additional markers from myocardial perfusion SPECT. J Biomed Res. 2013; 27: 467-477.

11 Hesse B, Tägil K, Cuocolo A, et al. EANM/ESC procedural guidelines for myocardial perfusion imaging in nuclear cardiology. Eur J Nucl Med Mol Imaging. 2005; 32: 855-897.

12 Sciagrà R. The expanding role of left ventricular functional assessment using gated myocardial perfusion SPECT: the supporting actor is stealing the scene. Eur J Nucl Med Mol Imaging. 2007; 34: 1107-1122.

13 Bonow RO. Gated myocardial perfusion imaging for measuring LV function. J Am Coll Cardiol. 1997; 30: 1649-1650.

14 Chen J, Garcia EV, Folks RD, et al. Onset of left ventricular mechanical contraction as determined by phase analysis of ECG-gated myocardial perfusion SPECT imaging: development of a diagnostic tool for assessment of cardiac mechanical dyssynchrony. J Nucl Cardiol. 2005; 12: 687-695.

15 Trimble MA, Borges-Neto S, Honeycutt EF, et al. Evaluation of mechanical dyssynchrony and myocardial perfusion using phase analysis of gated SPECT imaging in patients with left ventricular dysfunction. J Nucl Cardiol. 2008; 15: 663-670.

16 Driessen RS, Raijmakers PG, Stuijfzand WJ, Knaapen P. Myocardial perfusion imaging with PET. Int J Cardiovasc Imaging. 2017; 33: 1021-1031.

17 Lim SP, McArdle BA, Beanlands RS, Hessian RC. Myocardial viability: it is still alive. Semin Nucl Med. 2014; 44: 358-374.

18 Zdanowski P, Królicki L. The role of PET and CT examinations in the assessment of myocardial viability [in Polish]. Kardiol Pol. 2015; 73: 466-468.

19 Kobylecka M, Mazurek T, Królicki L. Evaluation of myocardial viability in PET [in Polish]. Kardiologia po Dyplomie. 2009; 5: 51-58.

20 Bax JJ, Wijns W, Cornel JH, et al. Accuracy of currently available techniques for prediction of functional recovery after revascularization in patients with left ventricular dysfunction due to chronic coronary artery disease: comparison of pooled data. J Am Coll Cardiol. 1997; 30: 1451-1460.

21 Löffler AI, Bourque JM. Coronary microvascular dysfunction, microvascular angina, and management. Curr Cardiol Rep. 2016; 18: 1.

22 Gould KL, Johnson NP, Bateman TM, et al. Anatomic versus physiologic assessment of coronary artery disease. Role of coronary flow reserve, fractional flow reserve, and positron emission tomography imaging in revascularization decision making. J Am Coll Cardiol. 2013; 62: 1639-1653.

23 Mayala HA, Bakari KH, Mkangala A, et al. The association of 18F-FDG PET/ $\mathrm{CT}$ and biomarkers in confirming coronary microvascular dysfunction. BMC ResNotes. 2018; 11: 796.

24 Brainin P, Frestad D, Prescott E. The prognostic value of coronary endothelial and microvascular dysfunction in subjects with normal or non-obstructive coronary artery disease: A systematic review and meta-analysis. Int J Cardiol. 2018; 254: 1-9.

25 Jarząbek K, Sobczyk A, Sobczyk W, et al. The concept of unstable atherosclerotic plaque and pharmacological therapeutic strategies. Polish Surgery. 2015; 17; 1-12.

26 Graebe M, Pedersen SF, Borgwardt L, et al. Molecular pathology in vulnerable carotid plaques: correlation with-18 fluorodeoxyglucose positron emission tomography (FDG-PET). Eur J Vasc Endovasc Surg. 2009; 37: 714-721.

27 Tarkin JM, Joshi FR, Evans NR, et al. Detection of atherosclerotic inflammation by 68Ga-DOTATATE PET compared to [18F]FDG PET imaging. J Am Coll Cardiol. 2017; 69: 1774-1791.

28 Dweck MR, Chow MW, Joshi NV, et al. Coronary arterial 18F-sodium fluoride uptake: a novel marker of plaque biology. J Am Coll Cardiol. 2012; 59: 1539-1548.

29 Derlin T, Richter U, Bannas P, et al. Feasibility of 18F-sodium fluoride PET/CT for imaging of atherosclerotic plaque. J Nucl Med. 2010; 51: 862-865.

30 Sachpekidis C, Sachpekidis V, Moralidis E, Arsos G. Equilibrium radionuclide ventriculography: still a clinically useful method for the assessment of cardiac function? Hell J Nucl Med. 2018; 21: 213-220.

31 Ahmad I, Amir N, Durr-E-Sabih, et al. Preparation and radiochemical control of $99 \mathrm{mTc}$ labeled blood pool agent for in vivo labelling of the red blood cells. Acta Pol Pharm. 2014: 71: $245-248$

32 Dae M, DeMarco T, Botvinick E, et al. Scintigraphic assessment of MIBG uptake in globally denervated human and canine hearts- implications for clinical studies. J Nucl Med. 1992; 33: 1444-1450.

33 Langer A, Freeman MR, Josse RG, Armstrong PW. I-123 Metaiodobenzylguanidine in diabetes mellitus: assessment of cardiac sympathetic denervation and its relation to autonomic dysfunction and silent myocardial ischemia. J Am Coll Cardiol. 1995; 25: 610-618.

34 Merlet $\mathrm{P}$, Vallette $\mathrm{H}$, Dubois $\mathrm{R}$, et al. Prognostic value of cardiac metaiodobenzylguanidine imaging in patients with heart failure. J Nucl Med. 1992; 33: 471-477.

35 Arimoto $\mathrm{T}$, Tada $\mathrm{H}$, Igarashi $\mathrm{M}$, et al. High washout rate of iodine-123-metaiodobenzylguanidine imaging predicts the outcome of catheter ablation of atrial fibrillation. J Cardiovasc Electrophysiol. 2011; 22: 1297-1304

36 Ulman M, Ząbek A, Małecka B, et al. The safety and utility of magnetic resonance imaging in a patient with conventional cardiac resynchronization therapy device. Pol Arch Intern Med. 2017; 127: 703-704.

37 Ostręga M, Gierlotka MJ, Słonka G, et al. Clinical characteristics, treatment, and prognosis of patients with ischemic and nonischemic acute severe heart failure. Analysis of data from the COMMIT-AHF registry. Pol Arch Intern Med. 2017; 127: 328-335.
38 Holcman K, Kostkiewicz M, Szot W, et al. Inflammatory parameters and results of scintigraphy with radiolabelled 99mTc-HMPAO leukocytes in patients with suspected infective endocarditis [in Polish]. Przegl Lek. 2017; 74; 302-305.

39 Kawińska-Hamala A, Kawiński J, Wranicz JK. The usefulness of positron emission tomography/computed tomography with 18F-fluorodeoxyglucose in the diagnosis of cardiac device-related infective endocarditis. Kardiol Pol. 2018; 76: 1379.

40 Domagała SJ, Domagała M, Chyła J, et al. Ten-year study of late electrotherapy complications. Single-centre analysis of indications and safety of transvenous leads extraction. Kardiol Pol. 2018; 76: 1350-1359.

41 Kouijzer IJ, Vos FJ, Janssen MJ, et al. The value of ${ }^{18} \mathrm{~F}-\mathrm{FDG}$ PET/CT in diagnosing infectious endocarditis. Eur J Nucl Med Mol Imaging. 2013; 40: 1102-1107.

42 Płońska-Gościniak E, Olędzki S, Kukulski T, et al. Pol-CDRIE registry - 1-year observational data on patients hospitalized due to cardiac device-related infective endocarditis in Polish referential cardiology centres. Kardiol Pol. 2019; 77: 561-567.

43 Bruun NE, Habib G, Thuny F, Sogaard P. Cardiac imaging in infectious endocarditis. Eur Heart J. 2014; 35: 624-632.

44 Ramirez R, Trivieri M, Fayad ZA, et al. Advanced imaging in cardiac sarcoidosis. J Nucl Med. 2019; 60: 892-898.

45 Tuominen H, Haarala A, Tikkakoski A, et al. FDG-PET in possible cardiac sarcoidosis right ventricular uptake and high total cardiac metabolic activity predict cardiovascular events. J Nucl Cardiol. 2019; 2: 1-7.

46 Holcman K, Kostkiewicz M, Podolec P, Rubiś P. Cardiac amyloidosis - state of the art. Diagnosis and emerging therapies. Folia Cardiologica. 2019; 14: 616-624. 47 Gillmore JD, Maurer MS, Falk RH, et al. Nonbiopsy diagnosis of cardiac transthyretin amyloidosis. Circulation. 2016; 133: 2404-2412.

48 Perugini E, Guidalotti PL, Salvi F, et al. Noninvasive etiologic diagnosis of cardiac amyloidosis using 99mTc-3,3-diphosphono-1,2-propanodicarboxylic acid scintigraphy. J Am Coll Cardiol. 2005; 46: 1076-1084.

49 Berman DS, Maddahi J, Tamarappoo BK, et al. Phase II safety and clinical comparison with single-photon emission computed tomography myocardial perfusion imaging for detection of coronary artery disease: fluorpiridaz $\mathrm{F} 18$ positron emission tomography. J Am Coll Cardiol. 2013; 61: 469-477.

50 Moody JB, Poitrasson-Rivière A, Hagio T, et al. Added value of myocardial blood flow using ${ }^{18} \mathrm{~F}$-flurpiridaz PET to diagnose coronary artery disease: the flurpiridaz 301 trial. J Nucl Cardiol. 2020 Jan 30. [Epub ahead of print].

51 Sinusas AJ. The potential of myocardial imaging with hypoxia markers. Semin Nucl Med. 1999; 29: 330-338.

52 Petkow-Dimitrow P, Tomkiewicz-Pająk L, Karpiński M, et al. Cardiac magnetic resonance imaging in a woman suspected of hypertrophic cardiomyopathy based on genotyping. Pol Arch Intern Med. 2018; 128: 617-618. 